\title{
Direct numerical simulation of two-phase gas dynamic flows with phase transition for water and for liquid sodium
}

\author{
Anna Aksenova ${ }^{1}$, Vladimir Chudanov ${ }^{1 *}$, Alexey Leonov ${ }^{2}$ and Artem Makarevich ${ }^{1}$ \\ ${ }^{1}$ IBRAE, Nuclear Safety Institute, 115191, Moscow, B. Tulskaya 52, Russia \\ ${ }^{2}$ MEPhi, National Research Nuclear University, 115409, Moscow, Kashirskoe highway 31, Russia
}

\begin{abstract}
In this paper the results of the calculation for one-dimensional problems with water, as well as, a two-dimensional calculation simulating an experimental study of heat transfer and resistance in the vertical pipe with boiling sodium subject to forced lift conditions are presented.
\end{abstract}

\section{Introduction}

There are two classes of methods to simulate two-phase flows with interface (boundary) between phases: 1) the interface is considered as a sharp break; 2) the interface is considered as a diffusion region, like contact discontinuities in the gas dynamics. Models that allow describing the diffusion regions are divided into nonequilibrium (there is no equality of phase pressures and phase velocities) [1] and equilibrium (phases are in mechanical equilibrium) [2]. Numerical approximation for equilibrium models proves to be simpler, but in this case a number of problems arises [3]: 1) the nonmonotonic behavior of a two-phase mixture sound velocity as a function of the volume fraction, that can lead to errors in the calculation of the passage of waves across the interface; 2) non-conservative form of the equation for the volume fraction. Even using of specially obtained relations on the front of the shock wave [4], it is not always possible to achieve a coincidence between the numerical and analytical solutions, since the averaging of non-conservative variables has no physical meaning. This problem was overcome in [3], using the relaxation algorithm instead of the averaging procedure. A more robust approach involves the model that is nonequilibrium only by pressure [5]. In this model dependence of the sound speed on the volume fraction turns out to be monotonous and equal to the "frozen" mixture sound speed. Such imaginary complication of the model leads to a simplification of the numerical algorithm [6]. The involving of the surface tension forces in such two-phase model is realized using the Hamiltonian principle of least action. As a result, in the equations for the momentum and total energy of the two-phase mixture the additional tensor components appear. These terms contain the second spatial derivatives of the mass fraction of the liquid phase [7]. In this paper, the non-equilibrium pressure model is used to calculate the twophase flows. This model takes into account the surface tension forces using modified relations at the shock front [8]. To simulate phase transition the equilibrium model [9] is

* Corresponding author: chud@ibrae.ac.ru 
used. In this model heat and mass transfer processes set the two-phase system into a state with the same values of pressures, temperatures, and Gibbs energies. As closure relations the equations of state "stiffened" $\left(e=\frac{p+\gamma \pi}{\rho(\gamma-1)}+q\right)$ or "Noble-Abel-Stiffened" $\left(e=\frac{(p+\gamma \pi) \times(1-\rho b)}{\rho(\gamma-1)}+q\right)$ are used. The "Noble-Abel-Stiffened" system, in addition to the attractive forces, allows taking into account the molecular repulsive forces [10], which can significantly improve the accuracy of direct numerical simulation. The constant parameters for the specified equation of state are found from the experimental saturation curves for the enthalpies, densities and pressure in the fixed range of the temperatures [11, 12]. Calculations related to the influence of capillary effects in two-phase flows are presented in [13]. In this paper the results of the calculation for one-dimensional problems with water, as well as, a two-dimensional calculation simulating an experimental study of heat transfer and resistance in the vertical pipe with boiling sodium subject to forced lift conditions are presented. In conclusion, the shortcomings of the presented technique for the direct numerical simulation of mass transfer and variants of it modification are indicated.

\section{Cavitation in the water}

The calculation of cavitation in the water was performed for the "stiffened" EOS with the coefficients presented in [14]. The tube with water of density $1150 \mathrm{~kg} / \mathrm{m}^{3}$ at atmospheric pressure is 1 meter long and the initial discontinuity is located at 0.5 meter. The liquid is assumed to contain a uniformly distributed small amount of vapor, $10^{-2}$ in the whole domain. The temperature of both phases is the same. The speed of the two-phase medium in the left part of the tube is equal $-2 \mathrm{~m} / \mathrm{s}$, and the velocity in the right-hand part of the pipe is equal to $2 \mathrm{~m} / \mathrm{s}$. In this calculation, an explicit interface between phases is initially absent, cavitation occurs in a two-phase mixture with a small fraction of the gas phase. The results of the cavitation calculation for the "stiffened" EOS (Table 1) using a grid of 4096 cells at time of $3.2 \mathrm{msec}$ are presented in Figure 1. The profiles of pressure (a) and speed (b) are shown. Each graph is obtained using the model described in this paper with taking to account of the mass transfer and without of one. The results of the presented calculations qualitatively coincide with the dependences obtained in [14] for the same problem.

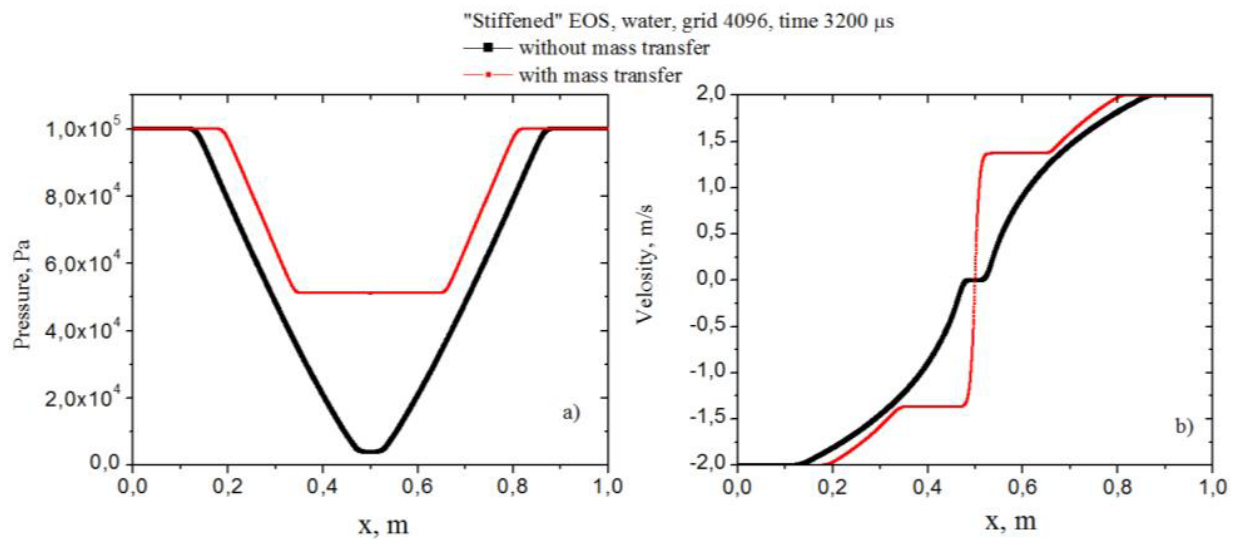

Fig. 1. Cavitation in the water. 
Table 1. "Stiffened" EOS coefficients for water [14].

\begin{tabular}{|c|c|c|c|c|c|}
\hline Phase & $\boldsymbol{\gamma}$ & $\boldsymbol{\pi}, \mathbf{P a}$ & $\mathbf{q}, \mathbf{J} / \mathbf{k g}$ & $\mathbf{q} \mathbf{}, \mathbf{J} / \mathbf{k g} / \mathbf{K}$ & $\mathbf{C}_{\mathbf{V}}, \mathbf{J} / \mathbf{k g} / \mathbf{K}$ \\
\hline Liquid & 2.35 & $1 \times 10^{9}$ & $-1167 \times 10^{3}$ & 0 & 1816 \\
\hline Gas & 1.43 & 0 & $2030 \times 10^{3}$ & -23400 & 1040 \\
\hline
\end{tabular}

\section{Simulation of 2D heat and mass transfer in a vertical channel with boiling sodium subject to forced lift conditions}

The vertical dimension of the working region of a flat channel with transverse dimension of $5 \mathrm{~mm}$ is $70 \mathrm{~cm}$. The heat flux through the side walls was assumed to be $\sim 0.57 \mathrm{MW} / \mathrm{m}^{2}$. The initial volume fraction of sodium vapor in the whole pipe is $10^{-5}$, and the initial temperature of two-phase sodium mixture was assumed equal to $1153 \mathrm{~K}$ everywhere, which is $3 \mathrm{~K}$ less than the boiling point at atmospheric pressure. The distribution of the initial vertical velocity corresponds to a mass flow of $166 \mathrm{~kg} / \mathrm{m}^{2} / \mathrm{s}$. At the lower boundary, constant values of temperature and volume fraction are maintained, equal to the initial values. A constant mass flow is also maintained at the lower boundary. At the upper boundary, atmospheric pressure is fixed. The calculation was carried out using the "Stiffened" EOS with the parameters shown in Table 2.

Table 2. "Stiffened" EOS coefficients for sodium.

\begin{tabular}{|c|c|c|c|c|c|}
\hline Phase & $\boldsymbol{\gamma}$ & $\boldsymbol{\pi}, \mathbf{P a}$ & $\mathbf{q}, \mathbf{J} / \mathbf{k g}$ & $\mathbf{q} \mathbf{\mathbf { \prime }}, \mathbf{J} / \mathbf{k g} / \mathbf{K}$ & $\mathbf{C}_{\mathbf{V}}, \mathbf{J} / \mathbf{k g} / \mathbf{K}$ \\
\hline Liquid & 1.48 & $3.67 \times 10^{8}$ & -265030 & 0 & 864 \\
\hline Gas & 1.49 & 0 & 2086460 & -4149 & 1690 \\
\hline
\end{tabular}

A vertical profile of the pressure averaged over the cross section at time instant $0.22 \mathrm{~s}$ is shown if Figure 2.

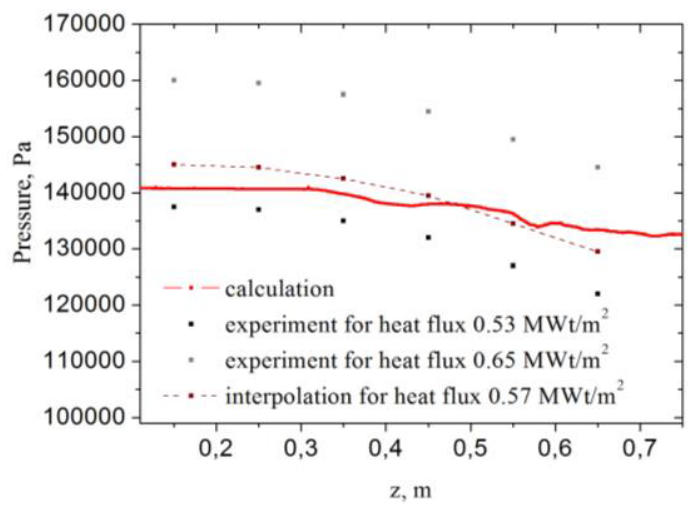

Fig. 2. Pressure distribution along the channel

The points correspond to the experimental values of the pressure for two different values of the heat fluxes [16]. The dashed curve corresponds to the interpolation of the experimental values for the heat flux of $0.57 \mathrm{MW} / \mathrm{m}^{2}$ assuming a linear dependence of the pressure values on the heat flux. A good qualitative agreement of the calculated and experimental values is obtained. The difference in the magnitude of the pressure difference over the working region of the pipe can be related to the "flat" approximation of the model calculation, which leads to different values of the ratio of the transverse length of the heating section to the enclosed area. 


\section{Conclusion}

The presented numerical method was constructed for 2D calculations of two phase mixture flows combining the noequilibrium pressure model approach [5] with conservative inclusion of surface tension forces using the Hamiltonian principle of least action [7] and with phase transition approach splitting heat and mass exchanges processes [9]. This numerical method is explicit and realized in parallel programming mode and allows one to obtain qualitative agreement with the experimental data. However, it should be noted that the "stiffness" of the non-linear system of equations in the equilibrium model provides not always an accurate simulation of mass transfer [14]. In such cases, the assumption of complete evaporation or condensation in the considered grid cell is applied [17]. Further development of a numerical technique for direct simulation of mass transfer in two-phase flows is associated with the use of a simplified relaxation solver, which ensures convergence to the same solutions as the conventional solver, but is much simpler and robust, since the equilibrium mass fraction is linearized after evaporation (or condensation) on the value of the equilibrium pressure [18]. It is necessary to mention that DNS is very sensitive tool for grid changing. The special study will be fulfilled for $2 \mathrm{D}$ computations using DNS and grid for this method.

\section{References}

1. R. Abgrall, R.Saurel, J. Comput. Phys. 186, 361 (2003)

2. R. Saurel, F. Petitpas, R. Abgrall, Fluid Mech. 607, 313 (2008)

3. F. Petitpas, E. Franquet, R. Saurel, O. Le Metayer, J. Comp. Phys. 225, 2214 (2007)

4. R. Saurel, O. Le Metayer, J. Massoni, S. Gavrilyuk, Shock Waves 16, 209 (2007)

5. R. Saurel, F. Petiptas, R.A. Berry, J. Comp. Phys. 228, 1678 (2009)

6. A. Leonov, V. Chudanov, A. Aksenova, Proc. IBRAE RAS, Issue 14, Moscow, Nauka, (2013)

7. R. Berry, R. Saurel, F. Petitpas, E. Daniel, O. Le Metayer, S. Gavrilyuk, N. Dovetta, R. Martineau, INL Report, Contract DE-AC07-05ID14517, October (2008).

8. A. Aksenova, A. Leonov, A. Makarevich, V. Chudanov, Proc. IBRAE RAS, Issue 16, Moscow (2016)

9. A. Zein, M. Hantke, G. Warnecke, J. Comput. Phys. 229, 2964 (2010)

10. R. Saurel, P. Boivinc, O. Le M'etayer, Computers \& Fluids (to be published), 2016

11. O. Le Metayer, J. Massoni, R. Saurel, Int. J. Therm. Sci. 43, 265 (2004)

12. O. Le Métayer, R. Saurel, Phys. Fluids 28, 4 (2016)

13. V. Chudanov, A. Aksenova, A. Leonov, Int. J. Math. Mod. Meth. App. Sci., 8, 248 (2014)

14. M. Pelanti, K.M. Shyue, J. Comput. Phys. 259, 331 (2014)

15. J. Fink, L. Leibowitz, Thermodynamic and transport properties of sodium liquid and vapor, ANL 9700, South Cass Avenue Argonne, Illinois, U.S.A., 60439 (1995)

16. Yu. Zeigarnik, V. Litvinov, High Temp. 5, 1116 (1977)

17. G. Faccanoni, S. Kokh, G. Allaire, Modelling and simulation of liquid-vapor phase transition in compressible flows based on thermodynamical equilibrium, ESAIM: M2AN 46, 1029 (2012)

18. A. Chiapolino, P. Boivin, R. Saurel, Int. J. Num. Meth. Fluids 83, 583 (2017) 